\title{
Verso la creazione di sistemi e sub-sistemi di difesa del Regno di Sardegna: piazzeforti, galere e prime torri nella prima metà del Cinquecento
}

\author{
Maria Grazia Rosaria Mele \\ CNR-Istituto di Storia dell’Europa Mediterranea, Cagliari, Italia, mele@isem.cnr.it
}

\begin{abstract}
The defense of the Kingdom of Sardinia with Fernando II of Aragon was based on control of urban fortifications and galley fleets from other realms of the Crown. In the conflict between Hispanic Monarchy, Turkish-Berber towns and Ottoman Empire is necessary to adapt the urban fortifications to modern needs and control territory. With Carlos V began to build the first coastal towers to protect ports and the mouths of major waterways. The galleys of Doria and the Carròs-Centelles ensured the mobile defense. A mid-sixteenth century, a project of a Franco-Ottoman attack showed the danger of losing the kingdom. Next to strengthen the city's defenses, other coastal towers to defend the territory formed real and specific sub-systems that materialize only from the eighties of the century.
\end{abstract}

Keywords: Mediterranean sea, modern age, defence, Sardinia, Hispanic Monarchy.

\section{Le incursioni}

Nei primi anni del Cinquecento, durante i viceregni di Joan Dusay, di Pedro Girón de Rebolledo e poi di Angel de Vilanova, la Sardegna fu più volte obiettivo delle scorrerie turco-barbaresche che videro Curtogoli ed i Barbarossa tra i maggiori protagonisti. La villa di Cabras, situata a Nord-Ovest della fertile piana alluvionale di Oristano, ricca di peschiere, fu la prima ad essere colpita e di cui si ha notizia nelle fonti documentarie conosciute. Lo stesso luogotenente del regno, i consiglieri di Oristano, gli ufficiali dei Campidani ed i vassalli di alcune encontradas interne all'isola, avevano l'obbligo di contribuire alla difesa di quella costa. Una flotta di sei galere del regno di Napoli, tra le quali una armata a spese del regno sardo e comandata dal Montbui, tentò inutilmente di limitare i danni di tali incursioni. Il primo novembre 1514, giorno di Tutti i Santi, fu attaccata la costa nord-orientale della Sardegna, nelle marine di Siniscola. Il 30 marzo 1515
Ferdinando nominò viceré di Sardegna Angel de Vilanova e gli consentì di requisire presso Valencia, Denia, de la Mata e Alicante qualsiasi tipo di imbarcazione gli fosse necessaria per il viaggio e per affrontare eventuali attacchi di pirati nei mari vicini alla Sardegna. Le istruzioni impartite al nuovo viceré e la documentazione della cancelleria di Ferdinando consentono di mettere in evidenza sia la preoccupazione costante da parte del Vilanova di informare regolarmente il sovrano, che una puntuale risposta del re ai dubbi del suo luogotenente. Il re Ferdinando ordinò, quindi, che si facessero i turni di guardia sulle marine e, pur consapevole che città e baroni non avrebbero visto di buon occhio questa soluzione perché avrebbe gravato sulle loro risorse, ribadì anche che due galere del regno di Napoli costeggiassero per qualche tempo 1'Isola, a patto però che il regno di Sardegna si impegnasse a mantenerle. La difesa costiera dei primi decenni del Cinquecento si 
basava sui punti chiave della difesa medioevale: la piazzaforte di Cagliari, capitale del regno, sulla quale si era intervenuti con i primi lavori di adeguamento, e quelle di Alghero, Castelsardo e Oristano, che avevano necessità di urgenti riparazioni.

Il 5 aprile 1515, pochi mesi prima di attaccare per la seconda volta la piazzaforte di Bugia, nell'agosto 1515, Heyreddin Barbarossa guidò una incursione che distrusse il villaggio di Uras, nella Sardegna centro-occidentale, deportando i suoi abitanti a Tunisi. Dei due fratelli fu con tutta probabilità quello minore a capitanare l'impresa, perché Oruç era convalescente a Tunisi. Un'epigrafe in sardo, ancora oggi murata nella chiesa di San Paolo di Sèrzela, attesta la totale distruzione del villaggio e costituisce una delle prime attestazioni della notorietà del Barbarossa con tale nome.

L'incursione dovette insistere su un insediamento già fortemente spopolato fin dal XIV secolo, come tutto il Bonorzuli e che solo nei primi anni del Seicento si provvide a ripopolare mediante il trasferimento di un centinaio di vassalli. Le coste dell'oristanese, come del resto gran parte del litorale sardo, erano ancora sguarnite di fortificazioni, fatta eccezione per le piazzeforti. Per un adeguamento alle nuove esigenze belliche e la creazione di bastioni si dovette attendere ancora qualche decennio, durante il regno di Carlo V d'Asburgo. La difesa mobile fu assicurata, invece, dalle flotte di galere che pattugliavano le acque del Mediterraneo, anche se si potè contare su una flotta del Regno di Sardegna solo nella prima metà del secolo XVII. Furono, questi, gli anni che videro il sardo muladí Hasan Agà, divenuto re di Algeri, difendere gli interessi delle città nordafricane quando di Heyreddin Barbarossa divenne ammiraglio della flotta ottomana. Tunisi, conquistata dal Barbarossa nel 1534, fu ripresa da Carlo V l'anno successivo con una impresa che vide coinvolte circa quattrocento imbarcazioni riunitesi nel Golfo di Cagliari. Nel 1541, mentre era diretto a recuperare Algeri, l'imperatore fece tappa ad Alghero per visitare lo stato dei lavori nelle opere di fortificazione, ma l'impresa nordafricana fu un insuccesso a causa del tempo inclemente. Come riportano le cronache, in tutte e due le occasioni fu Hasan Agà a difendere le due piazzeforti maghrebine dagli assedi delle truppe imperiali e fu sempre lui a rifornire di vettovaglie le imbarcazioni dei collaboratori del corsaro, caricandole di datteri, uva passa, olio, riso, vino di grano ed una bevanda a base di acqua, miele e spezie quando partì per effettuare l'ultima spedizione nel Mediterraneo.

La necessità di difendere un'isola, che costituiva un punto strategico di notevole importanza e al contempo avrebbe potuto garantire una certa risorsa economica, è chiaramente espressa anche in una lettera del vescovo di Ampurias, Ludovico de Cotes, un iberico che risedette in Sardegna a metà del Cinquecento: «es grande el daño que este reyno recibe por razón de no aver en él dos galeotas bien armadas... por que una fragata que viene con veinte turcos haze que no pesquen seys cientas barcas coral en el Alguer, que lo venden a precio de oro, y así en todas las otras partes de Cerdeña donde se pesca coral; $y$ se sabe por muy cierto que comiença un turco con una fragata y desde a dos años tiene tres galeotas...y assy está el reyno más pobre que puede haver en otra parte, pudiendo ser el más rico por razón de ser tan fertil y por estar una jornada por mar de Roma y dos de Genova y doze millas de Corçega y si fuesse tan cultivado como Sicília se sacaría del más trigo que de otra parte y mucha vitualla....» (AGS, Guerra y Marina, Leg. 55, 249).

Pur non essendo in prima linea come i presidi nordafricani, il Regno di Sardegna era un mondo di frontiera in cui tutto girava al rallentatore: regno fedele, ma più lento nel recepire le direttive della Corona e soprattutto soffocato dagli interessi dei ceti privilegiati, con un sottobosco che sicuramente lasciava molto spazio alle convenienze di ognuno. Assenteismo regio e lontananza del regno agevolavano la formazione di cordate baronali e di ceti emergenti, consentivano a numerosi esponenti della realtà locale di speculare sugli appalti regi, talvolta in contrasto con gli interessi e la tendenza accentratrice della Corona oppure servendosi delle prassi clientelari radicate nel territorio e nella Corte stessa. Un Regno di Sardegna che solamente negli anni Ottanta del 
Cinquecento riuscì ad istituire una Reale Amministrazione delle Torri e che dovette attendere la fine degli anni Trenta del XVII secolo per avere una sparuta flotta di galere per proteggere le sue coste. La Corona se ne assicurava il possesso mantenendo le piazzeforti di Cagliari, Alghero e Castellaragonese, lasciandolo perennemente esposto alle incursioni, che che si abbatterono sull'isola per tutto il secolo XVI ed in quelli successivi, indebolendone l'economia.

\section{Il pericolo di perdere il regno}

Ci furono però alcuni momenti in cui il regno corse il rischio di una conquista franco turca. Utilizzata dagli spagnoli come appoggio militare e scalo intermedio per lo spostamento dei tercios e per il rifornimento delle squadre navali, l'isola sarda fu al contempo oggetto di interessi commerciali e strategici da parte della Francia. Già dopo la perdita dell'isola di Rodi, nel 1522, secondo Giacomo Cappello Carlo V concepì l'idea di concedere la Sardegna all'Ordine di San Giovanni di Gerusalemme al posto della sede di Malta, che nel $1530 \mathrm{fu}$ accettata come definitiva. Nel frattempo, tra la fine del 1527 e gli inizi del 1528, in un clima di destabilizzazione dell'equilibrio italiano, la coalizione franco-genovese attaccò il settentrione dell'isola: Castellaragonese fu assediata e riuscì a resistere, mentre Sassari fu invasa.

Scampato anche il pericolo di una conquista francese nel giugno del 1535 , se in altre realtà si cercò di far tesoro dei momenti di tregua per provvedere al rafforzamento delle fortificazioni, in Sardegna il viceré Antonio de Cardona non riuscì con il suo operato a raggiungere del tutto gli effetti desiderati, e non solo in materia di difesa. Nell'isola si cominciarono a rafforzare con i bastioni alcune tradizionali piazzeforti isolane -adeguando le più importanti alle necessità belliche del tempo- ed a proteggere le attività economiche, erigendo le prime torri allo sbocco dei corsi d'acqua e degli scali portuali. È sufficiente vedere in sintesi quali furono gli interventi durante il parlamento convocato dal viceré Antonio de Cardona, negli anni Quaranta del secolo, per avere un'idea della situazione dopo il passaggio di Carlo V ad Alghero, nel suo viaggio verso Algeri. Principali destinatarie dei finanziamenti, peraltro non certo sufficienti per far fronte alla situazione, furono la capitale del regno e le città regie di Alghero e Castellaragonese. Ad Oristano e Bosa si cominciarono a costruire grosse torri gallardas a protezione dei porti, ma non ancora inserite di un sistema. La difesa mobile fu assicurata dalle galere dei Doria che controllavano la parte settentrionale dell'Isola mentre quelle del conte di Quirra e dei suoi fratelli avrebbero potuto proteggere i possedimenti feudali meridionali e la capitale del regno. Lo stesso conte di Quirra, Guillem Ramon Carròs-Centelles, chiese all'imperatore di essere nominato ammiraglio di una flotta sarda, ma non riuscì nel suo intento.

Un forte impulso alla costruzione di nuove fortificazioni, anche se non raggiunse in Sardegna risultati immediati, si ebbe negli anni Cinquanta del secolo XVI, che videro la necessità di adeguare "a la moderna" le piazzeforti isolane mediante la pianificazione di un ingegnere regio. Ancora una volta, l'azione della Corona si concentrava soprattutto sulle piazzeforti di Cagliari, Alghero e Castellaragonese, tralasciando quelle di Oristano e di Iglesias non ritenute prioritarie. Così, mentre Giovanni Battista Calvi fu incaricato di occuparsi delle fortificazioni baleariche, il cremonese Rocco Capellino si trattenne per un ventennio a disegnare progetti per la maggior parte delle piazzeforti isolane e una carta di tutta la Sardegna.

Negli anni Cinquanta del secolo, il regno, inserito nell'ampio progetto di difesa delle maggiori piazzeforti del Mediterraneo contro la minaccia turco-algerina e francese, ebbe una sorte simile a quella delle vicine Baleari ma fu più esposto ai giochi di equilibrio italiani.

Nella primavera del 1553, oltre ai fermenti antimperiali nella Penisola italiana, si unirono ulteriori complicazioni create dalla flotta francoturca che conquistò quasi tutte le piazzeforti corse, ad eccezione di Calvi, che rimase sotto il controllo dei Doria. La conquista quasi totale della Corsica, e soprattutto la presa di Bonifacio, 
mettevano in serio pericolo la vicina Sardegna, anche per l'alta percentuale di corsi che abitavano nel Nord dell'isola.

Il Parlamento convocato a Cagliari dal viceré de Heredía ebbe, quindi, tra gli obiettivi più importanti quello di stanziare $i$ fondi necessari per la difesa. Vista l'urgenza, i tre stamenti riuscirono a trovare un accordo assicurando un donativo piuttosto sostanzioso di centomila ducati d'oro. La politica di difesa condotta in quegli anni di pericolo appare evidente nelle richieste avanzate dai rappresentanti degli stamenti e nella necessità di proteggersi dal pericolo franco-turco proveniente dalla vicina Corsica e di far progettare le opere ad un ingegnere, di potenziare la cavalleria e gli armamenti.

Altri autori hanno posto in evidenza lo stato di emergenza vissuto dal regno in quegli anni e che si può leggere nelle lettere inviate dal viceré de Heredia alla Corona e negli atti parlamentari. Che la situazione fosse molto più critica e difficilmente assimilabile alle preoccupazioni e lagnanze degli anni precedenti, è reso ancora più evidente dal fatto che la Corona si decise a commissionare progetti di adeguamento anche per le fortificazioni che fino ad allora non erano state tenute in conto e che furono trascurate anche nei decenni successivi. Il progetto oristanese mai realizzato del Rocco Capellino fu sicuramente il frutto di una situazione di emergenza, che aveva ben altre giustificazioni che non fossero solo l'azione devastante delle incursioni di pirati e corsari all'economia del regno. Nell'urgenza dettata dagli avvenimenti degli anni Cinquanta, si sfruttava quanto ereditato dalle fortificazioni precedenti, provvedendo all'adeguamento delle piazzeforti principali, e al contempo si cominciava a mettere in pratica l'idea di creare dei sub-sistemi di torri costiere facenti capo a tali piazzeforti.

La preoccupazione della Corona fu tale da spingere il principe-re Filippo a scrivere al padre, nel giugno 1555, manifestando il pericolo in cui si trovava il regno di Sardegna in relazione all'alleanza franco-turca e alla possibilità che nell'isola si sollevassero fermenti di ribellione, provenienti dalla vicina Corsica, nel Sassarese e nei terreni feudali della principessa di Salerno, Isabella de Villamarí, moglie di Ferrante di San Severino, che sempre in quegli anni aveva complottato contro gli spagnoli nel regno di Napoli. Filippo propose al padre un diretto controllo del regno di Napoli tramite Fernando Álvarez de Toledo y Pimentel, duca d'Alba, e una pronta azione preventiva nella Sardegna settentrionale tramite un luogotenente del viceré, a quel tempo malato, che si occupasse delle questioni del Logudoro, riferendosi soprattutto ai possedimenti feudali appartenenti alla principessa di Salerno, cioè il territorio e la città di Bosa, incamerata subito dopo nel patrimonio della Corona e trasformata in città regia (1556).

Un memoriale rinvenuto tra $\mathrm{i}$ documenti dell'Archivo General de Simancas fa un po' di luce sui reali timori del viceré e sul perché di tanto allarme, per un pericolo che evidentemente andava molto più in là della distruzione della città di Terranova e che aveva nella stessa Sardegna i promotori di un piano che proponeva ai francesi la conquista dell'Isola. Il progetto nacque nell'ambiente ecclesiastico sassarese e avrebbe potuto trarre vantaggio da una congiuntura particolare: l'alleanza segreta tra il pontefice Paolo IV e la Francia, i ribelli corsi, gli instabili equilibri fra gli stati italiani, la coalizione franco-turca. Di tale progetto non se ne fece nulla forse perché basato su una contingenza talmente temporanea e instabile che non si crearono poi, effettivamente, le condizioni giuste per realizzarlo. Tuttavia, conoscere la situazione con gli occhi di un osservatore del tempo può essere utile ad aggiungere ulteriori elementi per comprendere quegli anni Cinquanta del secolo che sono stati ben definiti un periodo di transizione. Un periodo di mutamento che la Monarchia spagnola e il regno di Sardegna stavano vivendo in pieno su differenti livelli: di portata più generale, perché immediatamente a ridosso all'abdicazione di Carlo V, e regnicolo, perché sfruttava l'interregno determinato dalla infermità e poi decesso del viceré de Heredia, con l'onnipresenza del governatore Geronimo de Aragall a gestire gli interinati.

Il memoriale fu redatto a Napoli il 7 dicembre 1555 dal cavaliere di San Giovanni di 
Gerusalemme Angel de Centelles e rilasciato in presenza di Bernardino de Mendoza, capitano delle galere di sua maestà e a quel tempo viceré ad interim del regno di Napoli. L'autore del documento parrebbe essere un figlio naturale del più importante feudatario sardo, il conte di Quirra Guillem Ramón Carròs-Centelles, e nipote di Enric e Serafí de Centelles, armatori di galere per sua maestà e impegnati ad esportare grano dalla Sardegna alle piazze iberiche di Valencia e Barcelona. Una nota nel documento indica un progettato attacco alle Baleari che effettivamente prese di mira Mahón nel 1558. Del progetto sardo, invece, non se ne fece nulla e in parte lo vediamo anche tramite lo stesso memoriale: l'azione avrebbe dovuto contare su numerosi fattori, la flotta ottomana non sembrava molto propensa a seguire gli interessi francesi e per poter mettere in atto la conquista della Sardegna bisognava prima concludere quella della Corsica. Le trattative, invece, forse andarono avanti su fronte pontificio (i riferimenti all'accordo segreto tra il pontefice e la Francia) e su quello relativo a Sampiero Corso, ma di fatto, anche queste si rivelarono deludenti diversi anni dopo.

La tregua di Vaucelles, per quanto provvisoria, consentì agli Austria di frenare il corso degli eventi, mentre la flotta turca nel Mediterraneo si era già rivolta verso il Levante. I francoottomani non condussero a buon fine la conquista della Corsica e la situazione si prorogò fino agli anni Sessanta del secolo, quando, nel nuovo quadro della pace di Cateau Cambresis, si prospettò la possibilità di cedere le Baleari e la Sardegna al pretendente al trono di Navarra, ma in quel caso non se ne fece nulla.

In ogni caso, il memoriale in questione pone in risalto l'importanza strategica del regno di Sardegna nello scacchiere mediterraneo. Di fatto, non solo la Corsica e le Baleari, ma anche la Sardegna, erano a rischio di un attacco francoturco, in questo caso con la connivenza degli stati italiani partitari della Francia e della Curia pontificia, nonché dei ribelli corsi. Altro elemento che la Corona non sottovalutò, fu quello della congiura napoletana, che vedeva implicato il principe di Salerno e avrebbe potuto creare problemi anche in Sardegna all'interno dei possedimenti feudali della moglie di costui.

Nonostante il malcontento generale, la proposta si sarebbe potuta considerare poco avveduta se non avesse potuto contare su una situazione internazionale veramente delicata. Il tutto si inserisce nella politica condotta dal nuovo pontefice, mirante a rafforzare il suo potere ergendosi ad arbitro della situazione internazionale e quindi a destabilizzare l'equilibrio già assai precario nella questione italiana, con una Monarchia spagnola che aveva dovuto far fronte alla perdita temporanea dei presidi toscani, ad una congiura nel napoletano, e per via indiretta alla conquista della Corsica ottenuta grazie all'alleanza franco-turca.

La scarsità di risorse a disposizione della Monarchia ispanica ne rendevano sicuramente problematica la difesa ma al contempo ne scoraggiavano una eventuale conquista da parte franco-ottomana, perché non sarebbe stato semplice mantenerla. Di contro, una possibile cessione sarebbe stata possibile solo nell'ambito di accordi internazionali che avessero cercato di arginare il fronte turco-barbaresco.

Gli anni che seguirono videro un potenziamento delle difese cittadine e, subito dopo la battaglia di Lepanto e la caduta del forte de La Goulette, un abbandono della politica aggressiva dell'Impero ottomano per privilegiare un progressivo incremento di incursioni che fiaccassero le economie dei regni mediterranei della Corona. Per far fronte a questo problema, in ogni regno mediterraneo della Monarchia ispanica, e quindi anche in Sardegna, fu creato un sistema di torri costiere presenti nel territorio rurale che segnalassero il pericolo e ne difondessero l'allarme, consentendo alla popolazione di potersi rifugiare nelle località maggiormente a riparo dalle incursioni. Le torri proteggevano il territorio e le attività economiche (peschiere, saline, zone di pesca del corallo, tonnare etc.). Come si può vedere nelle cartine proposte in questa sede da Daniele Vacca, le torri del regno sardo non furono costruite tutte nello stesso periodo. L'intero sistema fu realizzato nel corso di diversi decenni: alle prime torri gagliarde costruite allo 
sbocco dei fiumi e dei porti seguì la protezione della costa algherese e bosana, nella costa nordoccidentale dell'isola, per il controllo delle zone di pesca del preziosissimo corallo; poi fu la volta delle torri delle coste dell'Oristanese a protezione delle attività economiche del Marchesato, che garantiva notevoli introiti alla Corona; nel contempo fu protetta la capitale del regno con un vero e proprio subsistema difensivo meridionale; il sistema si perfezionò nel corso dei decenni finali del Cinquecento ed $\mathrm{i}$ primi decenni del Seicento, con la realizzazione di un vero e proprio sistema di torri che si traguardassero fra loro e creassero una catena di controllo del territorio su gran parte della costa sarda.

\section{Conclusioni}

Nei primi tempi, le incursioni fiaccarono l'economia del regno di Sardegna e impoverirono la popolazione, in parte fatta schiava e deportata nei bagni delle città maghrebine, alle quali si cercò di far fronte per lo più con la difesa mobile e con le guardie costiere. Il regno nel frattempo era mantenuto attraverso le fortificazioni urbane di Cagliari, Alghero e Castelsardo, che furono potenziate e adeguate alle nuove esigenze belliche nel corso di tutto il secolo XVI ma ancor più a partire dagli anni Cinquanta dello stesso secolo, quando si fece più concreta la possibilità di conquista del regno da parte dell'alleanza franco-turca. Negli anni Settanta del secolo, venuta meno tale preoccupazione, si optò per una difesa statica meno costosa di quella mobile e dislocata su tutto il territorio, che garantisse una più capillare protezione delle attività economiche: un sistema di torri costiere che si traguardassero fra loro e proteggessero le città, creando in alcuni casi dei veri e propri subsistemi attorno alle piazzeforti principali o comunque suddiviso in sargentias o distretti che garantivano il pronto intervento in caso di pericolo. In alcuni casi, infatti, come per la costa oristanese, la Corona si mostrò più interessata a tutelare le attività economiche più che la realtà urbana.

\section{Note}

Fin dal 2005 (Anatra, Mele, Murgia, Serreli, 2008), l'Istituto di Storia dell'Europa Mediterranea del CNR, in collaborazione con il regista multimediale Francesco Casu e l'architetto Olindo Merone, promuove la valorizzazione delle torri costiere e delle testimonianze supersititi attraverso il progetto Torri multimediali, in cui la torre racconta se stessa e la storia del suo contesto attraverso la multimedialità. Sono stati già allestiti la Torre di Chia (Domus de Maria-CA), e il Museo Turcus e Morus (Gonnostramatza-OR).

\section{Reference}

Anatra B., (1984), Dall'unificazione aragonese ai Savoia, in Day J, Anatra B., Scaraffia L., La Sardegna medioevale e moderna (Storia d'Italia, X, G. Galasso coord.), Torino, pp. 365 e ss.

Anatra B., Manconi F., (1999), Sardegna, Spagna e Stati italiani nell'età di Filippo II, Atti del Convegno internazionale di Studi (Cagliari, 5-7 novembre 1998), Cagliari.

Anatra B., Manconi F., (2001), Sardegna, Spagna e Stati italiani nell'età di Carlo V, Roma.

Anatra B., Mele M.G., Murgia G., Serreli G. (coord.), (2008), «Contra Moros y Turcos». Politiche e sistemi di difesa degli Stati mediterranei della Corona di Spagna in Età Moderna, Atti del Convegno internazionale di Studi (Villasimius-Santa Maria Navarrese, 2005), Cagliari.

Bono S., (1964), I corsari barbareschi, Torino, ERI.

Braudel F., (1928), Les espagnols et l'Afrique du Nord, de 1492 à 1577, «Revue Africaine», 69, pp. 184-233, 351-428.

Bunes Ibarra M.Á. de, (1998), El Norte de África y los otomanos a principio del siglo XVI, in Omenaje al Profesor Carlos Posac Mon, II, Ceuta, Instituto de Estudios Ceutíes, pp. 113-123.

Bunes Ibarra M.Á. de, (2004), Los Barbarroja, corsarios del Mediterráneo, Madrid, Aldebarán.

Cámara Muñoz A., (1998), Fortificación y Ciudad en Los Reinos de Felipe II, Madrid. 
Cámara Muñoz A., (1999). Las fortificaciones y la defensa del Mediterráneo, in Felipe II y el Mediterráneo, E. Belenguer cebrià, vol. IV, La monarquía y los reinos, II, Madrid: Sociedad Estatal para la Conmemoración de los Centenarios de Felipe II y Carlos V, pp. 355-376.

Belenguer Cebrià E. (coord.), (1999), Felipe II y el Mediterráneo, Sociedad Estatal para la Conmemoración de los Centenarios de Felipe II y Carlos V, Madrid.

Cámara A., (1990-1991), Las torres del litoral en el reinado de Felipe II: una arquitectura para la defensa del territorio, «Espacio, Tiempo y Forma», 3, 1990, pp. 55-86 e 4, 1991, pp. 53-94.

Cámara Muñoz A., (1998), Fortificación y Ciudad en Los Reinos de Felipe II, Madrid.

Casu S., Dessì A., Turtas R., (1995), Le piazzeforti sarde durante il regno di Ferdinando il Cattolico (1479-1516), in XIV Congresso di Storia della Corona d'Aragona (Sassari-Alghero, 19-24 maggio 1990), vol. II, t. 1, Sassari, Delfino Editore, pp. 217-261.

Casu S., Dessì A., Turtas R., (1996), Le piazzeforti sarde durante il regno di Carlo V fino alla battaglia di Algeri, in "Congreso de Historia de la Corona de Aragón", Jaca 20-25 de septiembre de 1993, Actas, Tomo I, volume $3^{\circ}$, Zaragoza, pp. 30-64.

Díaz Borrás A.,(1990), L'estudi de la pirateria a través dels avisaments costaners. Replegament cristià i setge islàmic a la València de transició a la modernitat, in «Anuario de Estudios Medievales», 20, pp. 275-295.

García Arenal M., Bunes Ibarra M.Á. de, (1992), Los españoles y el Norte de Africa. Siglos XV-XVIII, Madrid.

Guia Marin Ll.J., Mele M.G.R., Tore G. (coord.) (2014), Identità e frontiere: politica, economia e società nel Mediterraneo (secoli XIV-XVIII), Franco Angeli, Milano.

Guidetti M. (coord.), (1989), Storia dei Sardi e della Sardegna, 3, L'età moderna dagli aragonesi alla fine del dominio spagnolo, Milano, Jaca Book.

Mafrici M., (1995), Mezzogiorno e pirateria nell'età moderna (secoli XVI-XVIII), ESI, Napoli.

Sánchez C.J. (coord), Las fortificaciones de Carlos V, , Madrid, 2000.

Cantù F., Visceglia M.A. (coord.), (2003), L'Italia di Carlo V. Guerra, religione e politica nel primo Cinquecento, Atti del Convegno internazionale di studi (Roma, 2001), Roma.

Manconi F., 2002, Il governo del regno di Sardegna al tempo dell'imperatore Carlo V, Magnum Edizioni-Libreria Koinè (Quaderni, 1), Sassari.

Manconi, F., (2010), La Sardegna al tempo degli Asburgo: secoli XVI-XVII, Il Maestrale, Nuoro.

Lotti L., Villari R. (coord.), 2004, Filippo e il Mediterraneo, Laterza, Roma-Bari.

Mele G., (2006), Raccolta di documenti editi e inediti per la Storia della Sardegna, vol. 7, Documenti sulla difesa militare della Sardegna in età spagnola, Sassari.

Mele G., (2000), Torri e cannoni. La difesa costiera in Sardegna nell'età moderna, Sassari.

Mele M.G., (2009), La frontiera mediterranea: $i$ Centelles tra interessi feudali e difesa del Regno di Sardegna, in M.G. Meloni e O. Schena (coord.), Sardegna e Mediterraneo tra Medioevo ed Età Moderna, Studi in onore di F.C. Casula, Cagliari-Genova, pp. 207-223.

Mele M.G., (2011), Il conte di Quirra e i suoi fratelli nella prima metà del Cinquecento, in S. Cossu ed., Mamma mia su Moro!, Dolianova, pp. 47-53 .

Mele M.G., I Fratelli Barbarossa e la Sardegna ai tempi di Ferdinando il Cattolico, atti del Seminario Excursus storico fino al 1861 e contributo della Sardegna alla nascita dello Stato Unitario (Villacidro, 2/5/2011), disponibile in http://www.provincia.mediocampidano.it/resources/cms/documents/20110506_PUB_Atti_Convegn o_Storia_di_Sardegna.pdf [28.06.2013]

Mele M.G., (2013), I Carròs-Centelles e la Sardegna ai tempi di Ferdinando II d'Aragona e di Carlo V d'Asburgo, in Elites urbane e organizzazione sociale in area mediterranea fra tardo Medioevo e prima Età Moderna, Atti del Seminario di Studi (Cagliari, 1-2/12/2011), M.G. Meloni coord., (Europa e Mediterraneo. Storia e immagini di una comunità internazionale, 28), Cagliari, CNRISEM, pp. 443-462. 
Oliva A.M., Schena O. (coord.), (1998), I Parlamenti dei viceré Giovanni Dusay e Ferdinando Girón de Rebolledo (1495, 1497, 1500, 1504-1511), a cura di, Acta Curiarum Regni Sardinie, 5, Cagliari, Consiglio Regionale della Sardegna, 1998.

Pardo Molero J.F., (2001), La defensa del imperio. Carlos V, Valencia y el Mediteráneo, Sociedad Estatal para la Conmemoración de los Centenarios de Felipe II y Carlos V, Madrid.

Pillosu E, Un inedito rapporto cinquecentesco sulla difesa costiera della Sardegna di Marco Antonio Camos, «Nuovo Bollettino Bibliografico Sardo», nn. 21-24 (1959) e n. 25 (1960).

Pirinu A., (2013), Il Disegno dei baluardi cinquecenteschi nell'opera dei fratelli Paleari Fratino : le piazzeforti della Sardegna, All'insegna del Giglio, Borgo S. Lorenzo (FI).

Rodríguez Salgado M.J., (1994), Metamorfosi di un impero. La politica asburgica da Carlo V a Filippo II (1551-1559), (Cultura e Storia, 10), Milano 1994.

Rubiera Mata M.J. (coord.), (2001), Carlos V. Los moriscos y el Islam, Congreso Internacional (Alicante, 20-25 de noviembre de 2000), , Madrid, Società Estatal para la Conmemoración de los Centenarios de Felipe II y Carlos V, Universidad de Alicante.

Sola Castaño E., (1988), Un Mediterraneo de piratas: corsarios, renegados y cautivos, Madrid, Tecnos.

Sola Castaño E., (1998), Corsarios o reyes. De la saga de los Barbarroja a Miguel de Cervantes, Alcalá de Henares, consultabile on line in http://www.archivodelafrontera.com/corsarios.htm (Ringrazio il Prof. E. Sola Castaño per avermi gentilmente messo a disposizione il testo).

Tenenti A., I corsari in Mediterraneo agli inizi del Cinquecento in «Rivista Storica Italiana», LXXII (1960), pp. 272-284.

Viganò M., (2004), «El Fratìn mi ynginiero». I Paleari Fratino di Morcote, ingegneri militari ticinesi in Spagna (XVI-XVII secolo), Bellinzona. 